\title{
Physical Activity and Nutrition INfluences In ageing (PANINI): consortium mission statement
}

\author{
Anna C. Whittaker ${ }^{1}$ (1) Massimo Delledonne ${ }^{2} \cdot$ Taija Finni $^{3} \cdot$ Paolo Garagnani $^{4} \cdot$ Carolyn Greig $^{1,5} \cdot$ Victor Kallen $^{6}$. \\ Katja Kokko $^{7}$ - Janet Lord ${ }^{5,8}$ • Andrea B. Maier,10 • Carel G. M. Meskers ${ }^{9}$. Nadine Correia Santos ${ }^{11}$. \\ Sarianna Sipila ${ }^{7}$ Janice L. Thompson ${ }^{1} \cdot$ Natal van Riel $^{12}$
}

Received: 31 May 2017 / Accepted: 10 August 2017 / Published online: 1 September 2017

(c) The Author(s) 2017. This article is an open access publication

Keywords Ageing - Biomarkers · Multidisciplinary · Nutrition $\cdot$ Physical activity $\cdot$ Standardised measures

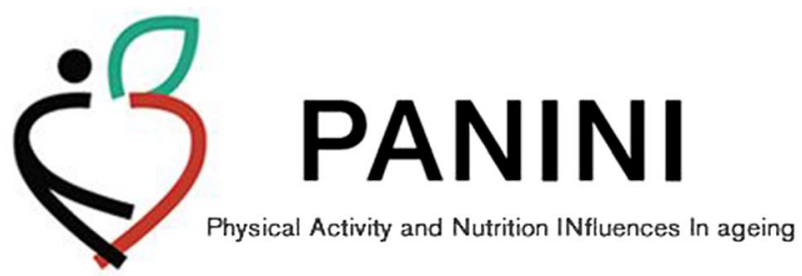

\section{Introduction}

Current demographic trends indicate that by the year 2020, almost one in five of the European population will be aged 65 years or over. Although life expectancy is increasing by 2 years per decade, the period of life spent in good health is not keeping pace and most Europeans spend their last

Anna C. Whittaker

a.c.whittaker@bham.ac.uk

1 School of Sport, Exercise and Rehabilitation Sciences, University of Birmingham, Birmingham, UK

2 Personal Genomics, University of Verona, Verona, Italy

3 Neuromuscular Research Center, Faculty of Sport and Health Sciences, University of Jyväskylä, Jyväskylä, Finland

4 Department of Experimental, Diagnostic, and Specialty Medicine (DIMES), University of Bologna, Bologna, Italy

5 MRC-Arthritis Research UK Centre for Musculoskeletal Ageing Research, University of Birmingham, Birmingham, UK

6 The Netherlands Organisation for Applied Scientific Research, The Hague, The Netherlands decade in poor health. Consequently, there is an urgent need to understand how lifestyle factors can influence age-related changes from gene to society level and how they may be integrated into a net effect of healthy ageing. It is also crucial to develop and validate interventions and health policies to ensure that more of our older adults have a healthy and active later life. This is an urgent and cross-cutting research priority in Europe, and to achieve this, it is vital to increase research capacity in this area to push forward the frontiers of scientific understanding. The Horizon 2020 funded Marie Curie Sklodowska Innovative Training Network-PANINI is addressing this capacity issue by focusing on research and training in two major interacting lifestyle factors with impact at multiple levels, namely, physical activity and nutrition.

The aims of PANINI are, therefore, to:

1. stimulate collaborative ageing research across Europe from the basic science to clinical intervention on the

7 Gerontology Research Center, University of Jyvaskyla, Jyväskylä, Finland

8 Institute of Inflammation and Ageing, Medical School, University of Birmingham, Birmingham, UK

9 Department of Human Movement Sciences, MOVE Research Institute Amsterdam VU University Amsterdam, Amsterdam, The Netherlands

10 Department of Medicine and Aged Care, Royal Melbourne Hospital, University of Melbourne, Melbourne, Australia

11 School of Medicine, University of Minho, Braga, Portugal

12 Department of Biomedical Engineering, Eindhoven University of Technology, Eindhoven, The Netherlands 
interaction of physical activity and nutrition through training a network of early stage researchers;

2. develop a standardised toolkit of the best practice measures of physical activity and nutrition;

3. utilise the toolkit across PANINI research projects to develop a shared data set across different European ageing populations;

4. coordinate existing data from collaborators to strengthen the PANINI data set to assess the physical activity and nutritional status interactions in a range of older adult populations;

5. compare novel physical activity and nutritional interventions to improve healthy ageing and assess the potential mechanisms underlying intervention responsiveness;

6. develop an online repository of training material on physical activity and nutrition in older adults for continuing professional development (CPD);

7. create a healthy ageing policy document with key stakeholders.

To promote healthy ageing, it is important to understand the mechanisms underlying unhealthy ageing trajectories. Healthy ageing is defined as optimising opportunities for good health, so that older people can take an active part in society and enjoy an independent and high quality of life (http://www.healthyageing.eu). Ageing is a complex process, but we are beginning to understand how age-related changes in physiological systems influence physical function. Good musculoskeletal function is critical for a healthy and active old age [1], but the musculoskeletal system is significantly affected by ageing, with loss of muscle mass and bone density from midlife resulting in increased physical frailty, falls, fractures, and loss of independence. The aetiology of sarcopaenia (low skeletal muscle mass and function) includes intrinsic factors associated with ageing, but more recently extrinsic modifiable lifestyle factors such as physical activity and nutrition have been suggested as primary drivers [2]. When associated with weight loss, fatigue, weakness, slow walking speed, and physical inactivity, this results in frailty [3]. Thus, early detection through comprehensive multidisciplinary assessment of a range of relevant factors and sensitive biomarkers, and mitigation through targeted interventions is essential [3].

Increased inactivity is a well-recorded feature of ageing humans, with less than half of those over the age of 50 years meeting the recommended guidelines for physical activity, and less than $10 \%$ in those over the age of 75 years. Furthermore, ageing is typically associated with a gain in fat mass (adiposity), which with decreased activity can contribute to sarcopaenia, and reduced mobility [4]. However, with ageing, energy intake also decreases, and malnutrition is prevalent [5], particularly in hospitalised patients and older outpatients [6]. Physical activity and optimal nutrition can influence the function of a range of body systems [7, $8]$, which interact in the maintenance of homeostasis and healthy ageing via enhancing quality of life, cognition, muscle mass, decrease fat mass, and modify sarcopaenia. However, data are sparse on energy-protein intake and energy expenditure in less fit older populations or communitydwelling older adults, where the prevalence of undernutrition may be far higher than currently assumed [7]. Consequently, nutritional and exercise requirements of elders to maintain physical and mental function are largely unknown, although the European Society for Clinical Nutrition and Metabolism (ESPEN) [9] have made evidence-based nutrition recommendations and the European College of Sport Science (ECSS) [10] have adapted existing physical activity guidelines for older adults. What is needed are multidimensional data over various populations, extending from midadulthood to old age, which PANINI will collect and analyse through development of its shared database and standardised toolkit of measures detailed below.

Understanding the biology and physiology underlying healthy/unhealthy ageing is an important initial step required before developing and testing physical activity and nutritional interventions. PANINI will address this through focusing on the impact of nutrition and physical activity on mobility, muscle function, endocrine function, cognitive function, and inflammation in ageing at the cell and system level. Dysregulation of mitochondrial function in the muscle is a hallmark of ageing and age- and lifestyle-related diseases and so will be studied in skeletal muscle, applying a system biology approach through the mathematical modelling of metabolism [11]. This will include assessment of the impact of diet and physical activity on metabolic activity across different age groups through the use of the newly generated PANINI data as well as existing data sets of PANINI collaborators, e.g., NU-AGE, MYOAGE, and MUMC+

Stress hormone changes with ageing relate to significant decrements in mental health and frailty, and increased inflammation, thus cortisol:dehydroepiandrosterone (DHEA) hormone ratios will be measured [12]. The decline in sex steroids in perimenopause can also have dramatic effects on muscle mass and function later on in life [13]. However, few studies have characterized changes in neuromuscular function, level of physical activity, and psychological characteristics in middle-aged women across menopausal stages. These biological and physiological changes will be studied within PANINI. Biological and physiological changes associated with ageing will be examined across a range of older populations of differing frailty phenotype, and novel interventions will be designed to reduce sedentary time, increase activity, and improve physical function. The study of sedentariness, and the development of interventions to reduce this will focus on older adults awaiting surgery and will utilise behaviour 
change techniques such as individualised feedback, individualised goal setting, and motivational interviewing, as well as environmental modification to encourage reduced sitting time. It will also utilise direct measurement of physical activity and sitting time variables to provide data on sitting time, standing time, stepping time, sit-to-stand transitions, and quantity of sedentary bouts $>30 \mathrm{~min}$. These data will be used to inform the design of a definitive trial to reduce sitting time prior to surgery, on selected health outcomes after surgery.

Further, ageing is often accompanied by an increase in systemic inflammation affecting physical and mental function [14], and dietary intake can also influence memory, attention, and learning [15]. Consequently, where possible within PANINI, cognitive function will also be assessed, and in particular, elderly cohorts, detailed food intake, and cognitive function will be assessed longitudinally to assess the impact of dietary choices and changes on cognitive decline. In addition, assessing dietary intake is challenging and subject to bias, but quantitative methods are cost- and labour intensive and only provide a narrow view of nutrients. PANINI will also use a mixed-methods approach that can provide a richer and broader view of food choice and eating behaviours. We will focus specifically on changes in social networks and their influence on dietary intake and physical function and will target under-studied, high-risk groups such as ethnic minorities, allowing us to design appropriate interventions that address barriers and enablers to sustained healthy food choices in old age across a range of populations.

The state of the art for interventions to improve musculoskeletal health is resistance training with/without nutritional supplementation [16], but efficacy is blunted in older adults, so innovative strategies to optimise responsiveness are needed. Physical activity interventions need to be appropriate for older adults across a range of health, frailty, and independence levels, and, as such, must be individually tailored. For example, major surgery induces stress, which combined with bed rest, can be harmful for patients, particularly when frail. Promoting a pro-physical activity and social environment before and during the entire surgical trajectory prevents frailty progression, and has been proven cost effective [17]. One of the barriers to physical activity in frail or recuperating populations is limited mobility and independence. There is evidence to support the effectiveness of chair-based muscle strengthening resistance exercise on muscle function [18]; however, it is not yet known how feasible these interventions are in very frail and dependent older adults, or how effective they are at altering key biomarkers of healthy ageing and well-being such as inflammation. PANINI will refine existing interventions by determining feasibility, effectiveness, and appropriate dose in a range of frail older adult populations including acute inpatients, sedentary older adults, care home residents, and hospital outpatients.

Discovering biomarkers of ageing are a crucial goal in ageing research. Epigenetics are the range of heritable reversible changes in chromatin structure and gene function, which can determine biological ageing and health status. Several candidate genes have been identified that show progressive increases in methylation across the human lifespan, and thus appear to be promising biomarkers $[19,20]$. However, further research is needed to determine whether gene methylation simply indicates chronological age or correlates with physiological function. Using this state-of-the-art technique, it is possible to develop interventions that could alter biological ageing via effects on epigenetic regulators, such as in FP7 project NU-AGE [21], focusing on the modulatory role of a modified Mediterranean diet on DNA methylation patterns during ageing [21, 22]. PANINI will expand this research by also examining methylation of candidate genes that relate to physiological function, and assess the impact of interventions on epigenetic modulation to provide further information on mechanisms of intervention effectiveness across several of the PANINI interventions. DNA methylation analysis of candidate biomarkers will be performed using Sequenom MassArray high-throughput platform and quantitatively analysed for DNA methylation using the EpiTYPER protocol. Candidate target regions will include genomic regions, whose methylation status are ageassociated and are informative of the biological age of an individual, according to published and proprietary data.

Finally, PANINI will explore the relevance of individual genetic background for the development of sarcopenic/frail phenotypes and for the success of nutritional and physical interventions. Studies of genetic determinants of muscle mass/strength have revealed different risk phenotypes for sarcopaenia [23-25]; however, an under-researched but relevant field is the relationship between nutritional habits and variants in genes that affect appetite and dietary preferences. PANINI will address this through comprehensively studying the interplay between genetic background, diet and sarcopenic/frail phenotype. Genetic analysis will stratify participants for their response to physical and nutritional interventions across PANINI. Next-generation sequencing (on a sub-sample) will integrate these results with the genetic characterizations in NU-AGE; candidate genomic variants will be evaluated on a larger mixed gender cohort. Metabolic network modelling will be used to study changes in energy metabolism associated with ageing. Model predictions will be validated with molecular data from a select number of participants in the PANINI data set. The main projects in PANINI and their key methodologies and outcome variables are summarised in Table 1. Publications as they arise will be open access and linked from the PANINI website http:// www.birmingham.ac.uk/panini. 


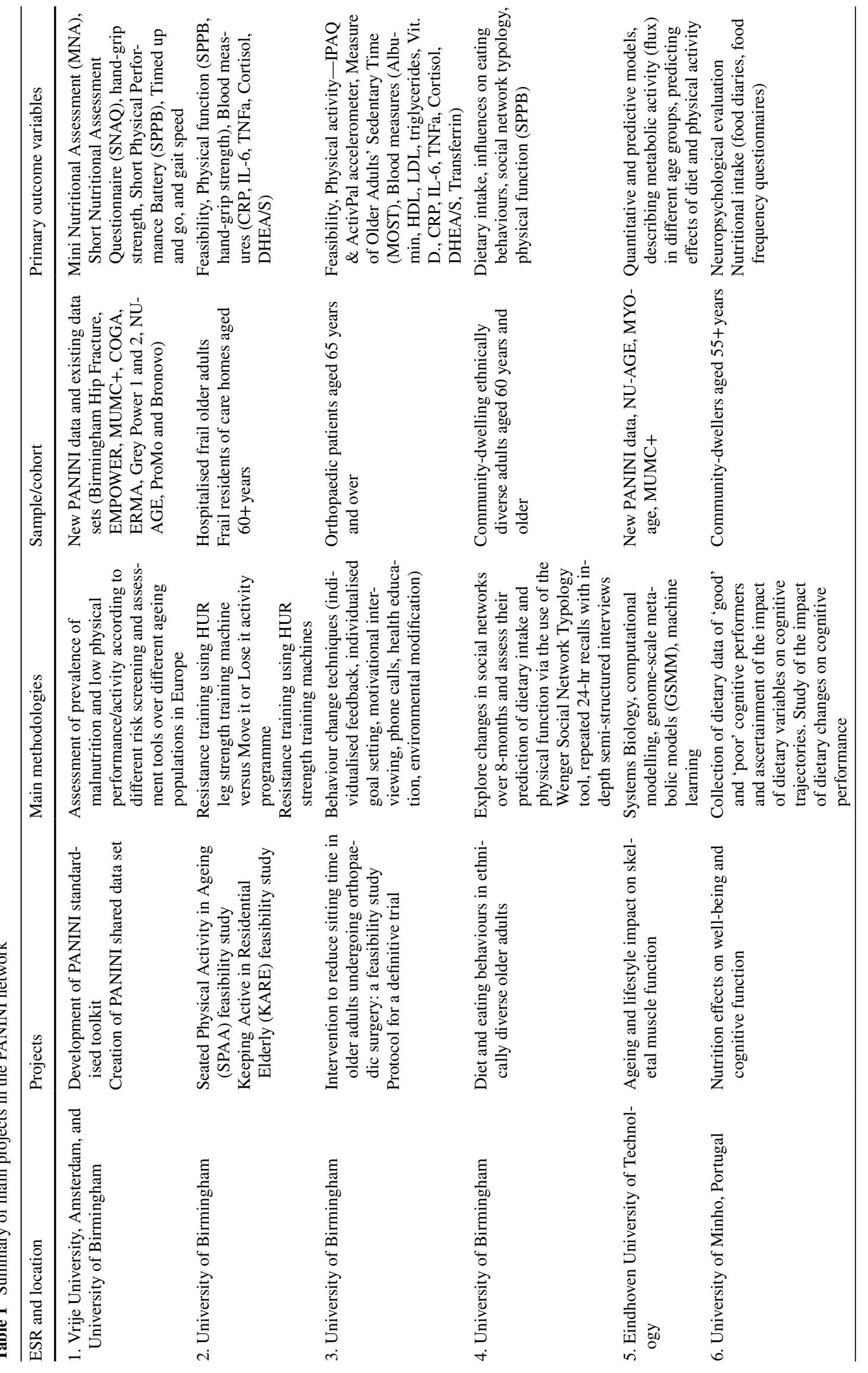




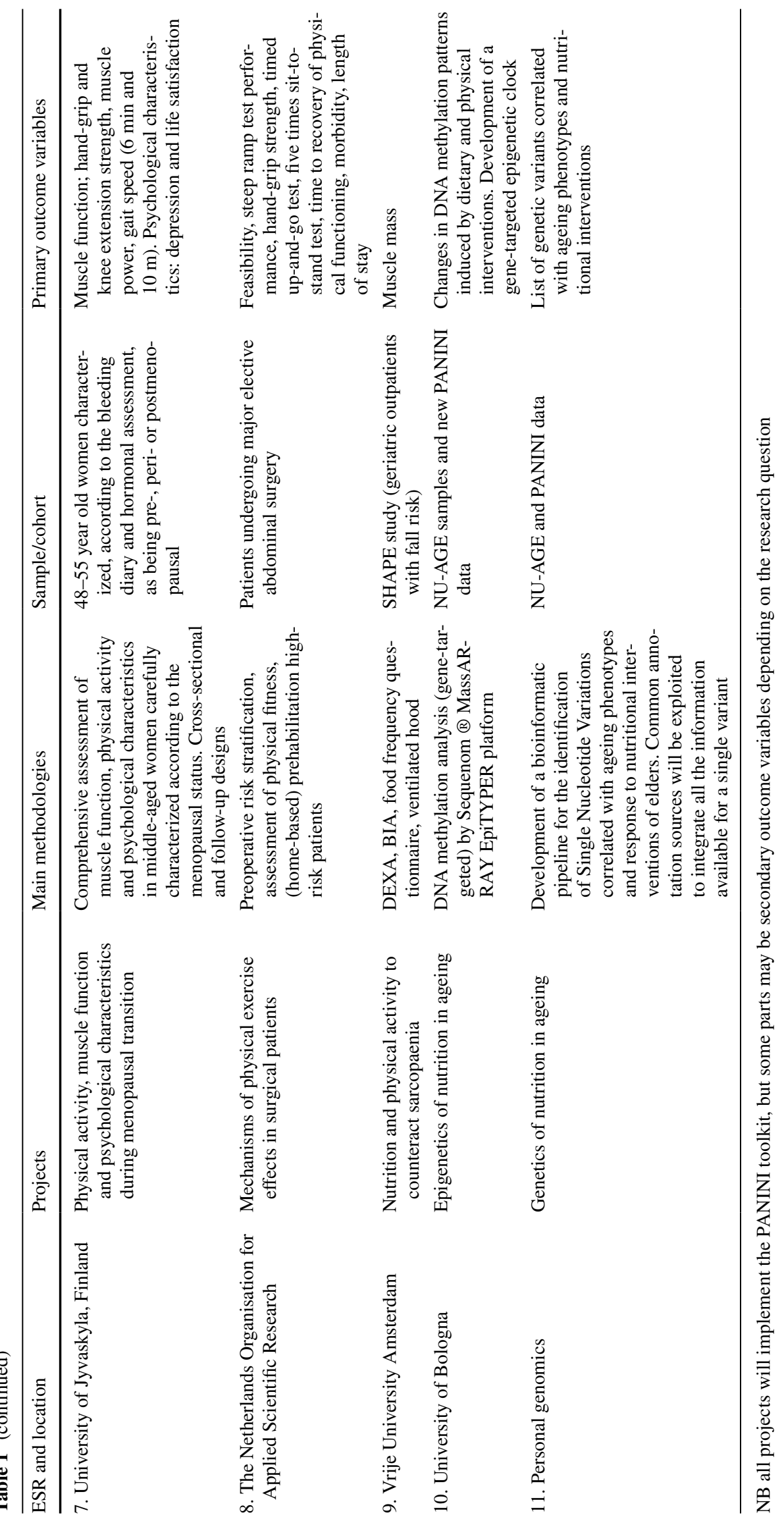




\section{The PANINI toolkit and shared data set}

PANINI will recommend a key set of gold standard nutrition and physical activity measures in ageing as a standardised toolkit, and make this widely available outside the PANINI consortium to encourage standardised measurement. Through the toolkit, we will bring together all of the PANINI projects to contribute to the development of a shared database for analysis of various ageing profiles across the range of older adult populations. Our data management plan (available on the PANINI website) is that this standardisation will result in a unique shared data set from which ageing profiles across countries, settings, frailty, and independence status could be determined and compared focusing particularly on nutritional status, physical activity, and physical function, as well as comparing relative change across the interventions in the network. The main components of the toolkit are: socio-demographics; anthropometrics (including body composition where possible); comorbidities and medication use; typical health behaviours including water intake; nutritional assessment questionnaire (and food frequency questionnaires or food/diet diaries where possible); physical activity via questionnaires (and accelerometry where possible); physical function via the short physical performance battery (SPPB); frailty through hand-grip strength, balance, and walking speed; falls efficacy and activities of daily living scales; brief cognitive assessment; and depression assessment.

We will also link, where possible, with matching data from existing European cohorts on ageing. For example, through the beneficiaries of the network and their existing links, we will collaborate to examine existing nutritional and physical function data in the Birmingham Hip Fracture, EMPOWER, MUMC+, COGA, ERMA, Grey Power 1 and 2, NU-AGE, ProMo, and Bronovo studies, with appropriate permissions. The PANINI shared data set will be made an open access resource at the conclusion of the project enabling the PANINI consortium and collaborators to answer research questions regarding physical activity and nutritional status impacts on healthy/unhealthy ageing. Researchers or networks wishing to become affiliated with the PANINI network for collaboration and/or data contributions to the shared data set should contact the Principal Investigator (PI)—Professor A. C. Whittaker.

\section{PANINI training}

To understand the impact of age-related changes upon normal body processes such as nutritional intake, and exercise capacity, and to relate this to overall effects on physical and mental well-being, a multidisciplinary approach is required. PANINI is providing multidisciplinary $\mathrm{PhD}$ training and secondments (summarised in Table 2) across an inter-sectoral network of eight leading academic and non-academic research institutions across Europe (six universities, one research enterprise, and one SME, as detailed in Table 1). Our partners: six private sector companies (Danone Nutricia Research; HUR Ltd.; HURLabs; Move it or Lose it; Scriptoria; and Blueberry Training); one large healthcare partner (University Hospitals Birmingham NHS Foundation Trust); two ageing charities (Age UK and UNIEKBO); and one Joint Research Council, who contribute to training, supervision, and dissemination, providing an innovative and inter-sectoral environment to produce maximum impact of PANINI's research. We are keen to open up the PANINI training network to other research and training networks; our main activities, open to (a limited number of) external participants, are detailed in Table 2; so please contact the PI if interested. It is also our plan to develop a CPD distance learning course from the materials developed and delivered during the PANINI project, after its completion, to increase training resources and build research capacity in multidisciplinary ageing research.

\section{Outreach, dissemination, and impact}

PANINI's approach to dissemination, impact, and innovation is to co-create our strategy with our stakeholders, holding public engagement events annually. These will allow us to refine our research methodology and dissemination approaches to meet stakeholder needs and expectations. Researchers will engage the public via annual individual or small group interactive activities such as science association events, older adults' societies, articles in older adults' newsletters, pod/vodcasts, and the media in a widely accessible manner. Further, an initial PANINI public engagement event is planned for midway through the project, where the ESRs will engage older adults with key messages and demonstrations from their projects. A final larger scale public engagement event is planned for the end of the network. Older adults and representatives from charities, industry, and the health sector with an interest in ageing research will be invited to attend these events. Midway through the project, it is our intention to present PANINI symposia at relevant interdisciplinary conferences with a focus on ageing, presenting the goals of the network and initial findings from the individual ESR projects and their collaborative work. Towards the end of the project, with key stakeholders, e.g., ageing charities, health professionals, and policy makers, we will refine a set of physical activity and nutritional intake recommendations for older adults of different frailty and dependency statuses which will be integrated with the knowledge gained regarding nutritional intake and physical function across the network. This will be integrated into 
Table 2 Main PANINI activities

\begin{tabular}{|c|c|}
\hline Activity & Month (from Jan 2016) \\
\hline Kick-off meeting & 2 \\
\hline Launch PANINI website and social media & 3 \\
\hline Data management plan finalised & 6 \\
\hline Standardised measures toolkit developed & 9 \\
\hline Standardisation: Shared database set up & 14 \\
\hline $\begin{array}{l}\text { ATC1: Biology of Ageing; Theories of Ageing; Musculoskeletal ageing; Brain ageing and cognition; Obesity, metabo- } \\
\text { lism and ageing }\end{array}$ & $14 *$ \\
\hline $\begin{array}{l}\text { ATC2: Physical activity and ageing; Sarcopaenia and exercise effects on muscle; Physical activity interventions for } \\
\text { healthy ageing }\end{array}$ & $14 *$ \\
\hline $\begin{array}{l}\text { ATC3: Nutrition and ageing; Genetics and longevity; Nutritional intake and protein-energy balance; Dietary patterns } \\
\text { and ethnic and cultural variations }\end{array}$ & $14 *$ \\
\hline Network meeting: ESR Project presentations & $14 *$ \\
\hline ESRs individual public engagement projects & Annually \\
\hline ATC4: Outreach \& dissemination. Scientific communication for non-specialists (Scriptoria) & $14 *$ \\
\hline $\begin{array}{l}\text { Group Secondment 1: Healthcare setting (University Hospitals Birmingham) New hospital design; Older people in } \\
\text { Hospital: Reminiscence, Design, Feedback, Listening to patients; Older people medicine: The in-patient experience, } \\
\text { Treating the older person; Nutrition: General health and well-being, Eating to stay healthy, Nutritional care after } \\
\text { trauma, Medication and nutrition; Post Stroke Care: The swallow reflex, Speech, Facial asymmetry, Mobility; Falls: } \\
\text { Prevention of harm, Fracture management, Rehabilitation, Returning home }\end{array}$ & 25 \\
\hline Public engagement event: Stakeholder involvement & $25^{*}$ \\
\hline $\begin{array}{l}\text { ATC5: Translation of research into product - (1) Physical activity research into intervention product development } \\
\text { (Move it or lose it); (2) How to translate genetic/epigenetic biomarkers research into a commercial tool (Personal } \\
\text { Genomics) }\end{array}$ & $25^{*}$ \\
\hline Visiting Scientist visit & $25^{*}$ \\
\hline Network meeting: ESR Project presentations & $25^{*}$ \\
\hline $\begin{array}{l}\text { ATC6: Business engagement. Formulating a business model towards a sustainable products and services portfolio; } \\
\text { Formulating a business case for innovations; Enhancing readiness towards investors and venture capital; Protecting } \\
\text { the IP portfolio (TNO) }\end{array}$ & $35^{*}$ \\
\hline $\begin{array}{l}\text { ATC7: Enterprise training. Creativity \& enterprise skills and the translation of research activity into products, patents } \\
\text { and spin-off companies (Blueberry Training) }\end{array}$ & $35^{*}$ \\
\hline $\begin{array}{l}\text { Group Secondment 2: Industry and technology. technology development and innovation for physical function assess- } \\
\text { ment (HUR \& HURLabs); technology development for nutrition delivery to vulnerable older adults (Danone Nutricia } \\
\text { Research); training and experience in innovation in patients' self-health assessments \& the Better in Better out } \\
\text { concept (TNO) }\end{array}$ & 35 \\
\hline ESR podcasts disseminated & 42 \\
\hline PANINI symposia at interdisciplinary international conference(s) - dissemination of scientific progress & By 42 \\
\hline Network conference & $44 *$ \\
\hline $\begin{array}{l}\text { ATC8: Getting research into health policy. Health Policy Legislation by the Healthy Ageing Commission Chair and UK } \\
\text { Chief Inspector of GPs; EU legislative framework: how policy works at EU level; EC vs. member states competencies } \\
\text { in health policy making (Age UK, UNIEKBO) }\end{array}$ & $44 *$ \\
\hline $\begin{array}{l}\text { ATC9: Health Policy document development - Introduction on the policy cycle (PC): To discuss how research is } \\
\text { needed in each phase of the PC; Case study: How does the JRC support the EC in policy making? Turning research } \\
\text { documents into policy-ready information; Discussion: How will your research support policy making in your member } \\
\text { state? (JRC) }\end{array}$ & 44 \\
\hline Network outreach and dissemination event & $44 *$ \\
\hline Develop CPD distance learning course based on PANINI materials & 45 onwards \\
\hline Make PANINI toolkit and shared database open access & 56 onwards \\
\hline
\end{tabular}

ATC advanced training course, ESR early stage researcher

* Limited spaces open to other researchers/networks, contact the PI A.C. Whittaker

a policy document for healthy ageing and discussed with representatives of health policy-making bodies at national (e.g., Public Health England) and European levels (e.g.,
EIP-AHA and DG-SANCO). These activities are summarised in Table 2; our dissemination plan is available on the PANINI website. 
In conclusion, a key target of PANINI is to develop a cohort of the next-generation researchers able to communicate healthy ageing research across disciplinary boundaries and value the benefits of the cross fertilisation of ideas and problem solving across the emerging interdisciplinary areas essential for integrative 'whole person' physical activity and nutrition research. Key impact will be through the anticipated widely disseminated significant advances in understanding the central processes that contribute to healthy ageing and the factors and mechanisms underpinning successful interventions. Key outputs will be a PANINI toolkit of recommended measures; a shared data set used to interrogate health and well-being in ageing across a range of older populations as predicted by the toolkit and a co-created health policy statement. Further, the training resources will also be made widely available in order as a resource to develop research capacity in physical activity and nutrition in ageing.

\section{Compliance with ethical standards}

Funding This project has received funding from the European Union's Horizon 2020 research and innovation programme under the Marie Skłodowska-Curie Grant Agreement No. 675003. http://www. birmingham.ac.uk/panini.

Conflict of interest The authors have no conflicts of interest.

Human and animal rights Human rights will be respected and all research is conducted in line with best ethical standards according to the Declaration of Helsinki. Animals are not being used in this project.

Informed consent All participants will provide informed written consent.

Open Access This article is distributed under the terms of the Creative Commons Attribution 4.0 International License (http://creativecommons.org/licenses/by/4.0/), which permits unrestricted use, distribution, and reproduction in any medium, provided you give appropriate credit to the original author(s) and the source, provide a link to the Creative Commons license, and indicate if changes were made.

\section{References}

1. Rantanen T, Guralnik JM, Foley D et al (1999) Midlife hand grip strength as a predictor of old age disability. JAMA 281:558-560

2. Narici MV, Maffulli N (2010) Sarcopaenia: characteristics, mechanisms and functional significance. Br Med Bull 95:139-159. doi:10.1093/bmb/ldq008

3. Fried LP, Tangen CM, Walston J et al (2001) Frailty in older adults: evidence for a phenotype. J Gerontol A Biol Sci Med Sci 56:M146-156

4. Zamboni M, Mazzali G, Fantin F et al (2008) Sarcopenic obesity: a new category of obesity in the elderly. Nutr Metab Cardiovasc Dis 18:388-395. doi:10.1016/j.numecd.2007.10.002

5. Verlaan S, Ligthart-Melis GC, Wijers SLJ et al (2017) High prevalence of physical frailty among community-dwelling malnourished older adults - a systematic review and meta-analysis. J Am Med Dir Assoc 18:374-382. doi:10.1016/j.jamda.2016.12.074
6. Pierik VD, Meskers CGM, Van Ancum JM et al (2017) High risk of malnutrition is associated with low muscle mass in older hospitalized patients-a prospective cohort study. BMC Geriatr 17:118. doi:10.1186/s12877-017-0505-5

7. Schilp J, Kruizenga HM, Wijnhoven HA et al (2012) High prevalence of undernutrition in Dutch community-dwelling older individuals. Nutrition 28:1151-1156. doi:10.1016/j.nut.2012.02.016

8. Bijlsma AY, Meskers CG, van den Eshof N et al (2014) Diagnostic criteria for sarcopaenia and physical performance. Age (Dordr) 36:275-285. doi:10.1007/s11357-013-9556-5

9. Deutz NE, Bauer JM, Barazzoni R et al (2014) Protein intake and exercise for optimal muscle function with aging: recommendations from the ESPEN Expert Group. Clin Nutr 33:929-936. doi:10.1016/j.clnu.2014.04.007

10. Biddle SJH, Brehm W, Verheijden M et al (2012) Population physical activity behaviour change: a review for the European College of Sport Science. Eur J Sport Sci 12:367-383

11. van Beek JH, Kirkwood TB, Bassingthwaighte JB (2016) Understanding the physiology of the ageing individual: computational modelling of changes in metabolism and endurance. Interface Focus 6:20150079. doi:10.1098/rsfs.2015.0079

12. Walston J, Hadley EC, Ferrucci L et al (2006) Research agenda for frailty in older adults: toward a better understanding of physiology and etiology: summary from the American Geriatrics Society/ National Institute on Aging Research Conference on Frailty in Older Adults. J Am Geriatr Soc 54:991-1001

13. Sipila S, Taaffe DR, Cheng S et al (2001) Effects of hormone replacement therapy and high-impact physical exercise on skeletal muscle in post-menopausal women: a randomized placebocontrolled study. Clin Sci (Lond) 101:147-157

14. Trollor JN, Smith E, Baune BT et al (2010) Systemic inflammation is associated with MCI and its subtypes: the Sydney Memory and Aging Study. Dement Geriatr Cogn Disord 30:569-578. doi:10.1159/000322092

15. van de Rest O, Berendsen AA, Haveman-Nies A et al (2015) Dietary patterns, cognitive decline, and dementia: a systematic review. Adv Nutr 6:154-168. doi:10.3945/an.114.007617

16. Pillard F, Laoudj-Chenivesse D, Carnac G et al (2011) Physical activity and sarcopaenia. Clin Geriatr Med 27:449-470. doi:10.1016/j.cger.2011.03.009

17. Hulzebos EHJ, van Meeteren NLU (2015) Making the elderly fit for surgery. Br J Surg 103:e12-e15

18. Fisher JA, Steele J, McKinnon P et al. (2014) Strength gains as a result of brief, infrequent exercise in older adults. J Sports Med 2014:731890

19. Garagnani P, Bacalini MG, Pirazzini C et al (2012) Methylation of ELOVL2 gene as a new epigenetic marker of age. Aging Cell 11:1132-1134. doi:10.1111/acel.12005

20. Burkle A, Grune T, Gonos ES et al (2015) Special issue biomarkers of human ageing. Mech Ageing Dev 151:1-1

21. Santoro A, Brigidi P, Gonos ES et al (2014) Mediterranean diet and inflammaging in the elderly: the European project NU-AGE. Preface. Mech Ageing Dev 136:1-2

22. Bacalini MG, Friso S, Olivieri F et al (2014) Present and future of anti-ageing epigenetic diets. Mech Ageing Dev 136:101-115

23. Walsh S, Ludlow AT, Metter EJ et al (2016) Replication study of the vitamin D receptor (VDR) genotype association with skeletal muscle traits and sarcopaenia. Aging Clin Exp Res 28:435-442

24. Di Renzo L, Gratteri S, Sarlo F et al (2014) Individually tailored screening of susceptibility to sarcopaenia using p53 codon 72 polymorphism, phenotypes, and conventional risk factors. Dis Mark. doi:10.1155/2014/743634

25. Tan LJ, Liu SL, Lei SF et al (2012) Molecular genetic studies of gene identification for sarcopaenia. Hum Genet 131:1-31 